\title{
Improving ROCs of Constant False Alarm Coded Anti-collision Radar in Very Noised Cases
}

\author{
Jamal Zaidouni, Atika Menhaj, Kamal Ghoumid, Slimane Mekaoui, Ali El Moussati, and Yassin El Hillali
}

\begin{abstract}
The use of Constant False Alarm Coded Anticollision Radar (CFACAR) is very interesting in automotive environment. Due to the orthogonality properties of used codes this system is most robust to multi-user interferences. The actual version of the receiver called in this paper Single Correlation Receiver (SCR), is not able to detect the targets in very low input Signal to Noise Ratio $(S N R)$. To resolve this problem, we present a new receiver called Averaging Correlation Receiver (ACR), that computes the average of the $M$ later correlations. Then, we developed the expression of detection and false alarm probabilities for the new receiver in mono and multi-user scenarios. These probabilities are used to plot the new Receiver Operating Characteristics (ROCs). They are drawn for different values of input $S N R$ and length $M$ of ACR. There is a suitable value of $M$, according to some equation, that can be taken to have a good detection (ROCs more perfect). Also, we found that for a fixed $S N R$, we must increase sufficiently the length $M$ but it is possible only for low relative velocity of the target. For a velocity of $5 \mathrm{Km} / \mathrm{h}$ with $M=1055$, we can lessen the value of the $S N R$ until we reach $S N R=-45 d B$.
\end{abstract}

Keywords-Automotive radar, Anti-collision, Detection, ROC, False alarm, Correlation Receiver

\section{INTRODUCTION}

C OLLISION avoidance systems are useful for automobile safety system to reduce the number of traffic accidents. These systems either provide a warning to the driver when there is an imminent collision or take action autonomously without any driver action. Many automotive manufacturers have developed in the last years systems based on radars or other type of sensors. For radars based systems, different techniques are used to estimate the distance and velocity of a potential obstacles. By applying some algorithms, we can ensure the safety distance and collision advance [1], [2], [3], [4]. The warnings given by the system should result in a minimum load on driver attention. Frequent warnings may desensitize the driver and cause future warnings to be ignored. Rare warnings can distract the driver during critical situations. Therefore, the method of warning the driver and the frequency at which warnings are given must be chosen carefully.

Bistatic and multistatic antenna systems have become increasingly popular for through-wall radar imaging because of their ability to collect more target scattering information.

J. Zaidouni, K. Ghoumid and A. El Moussati are with Department Electronics, Informatics and Telecommunications, École Nationale des Sciences Appliquées, ENSAO, Morocco (e-mail: jamal.zaidouni@gmail.com, ghoumid_kamal@yahoo.fr, a.elmoussati@ump.ac.ma).

A. Menhaj and Y. El Hillali are with Institut dÉlectronique de Microelectronique et de Nanotechnologie, University of Valenciennes, France (e-mail: atika.menhaj@univ-valenciennes.fr, yassin.elhillali@gmail.com).

S. Mekaoui is with Department Informatics and Telecommunications, Univ Alger, Algeria (e-mail: smekaoui@yahoo.fr).
To directly compare the abilities of bistatic and multistatic systems over monostatic radar alone, both human and clutter target data were measured and used to create ROC curves [5], [6].

The authors of [7], [8] have realized the first Compressive Sensing (CS) adaptive radar detector. They have proposed three novel architectures and have demonstrated how a classical Constant False Alarm Rate (CFAR) detector can be combined with 11-norm minimization. Using asymptotic arguments and the Complex Approximate Message Passing (CAMP) algorithm they have characterized the statistics of the 11-norm reconstruction error and have derived closed form expressions for both the detection and false alarm probabilities.

Target detection is one of the important functions of radar systems. In [9], [10], the authors have presented a detection method using total correlation (TC) based on information theory for noise radar systems which enables the detection of multiple targets at intermediate and low signal-to-noise ratio (SNR) regimes. The proposed method utilizes the largest eigenvalue of the sample covariance matrix to extract information from the transmitted signal replica, and outperforms the conventional TC detector when reflected signals have intermediate or low SNR values.

Based on the combination of time domain averaging and correlation, [11] proposed an effective time domain averaging and correlation based spectrum sensing (TDA-C-SS) method used in very low SNR environments. With the assumption that the received signal samples from the primary users are deterministic, the proposed TDA-C-SS method processes the received samples by a time averaging operation to improve the SNR.

The ultra-wide-band (UWB) technique is used in [12], [13] to develop the random noise radar (such as our system) for through-wall surveillance applications. They also discussed the phenomena of interference level and radar cross section (RCS) of the human target using the ROCs. Moreover, the [14] has presented an application of UWB radar in robot localization and site recognition.

In [15], [16], they have presented a signal processing technique for Frequency Modulated Continuous Wave (FMCW) radar sensors. They have used chirp sequence modulation which allows the observation of slow-moving obstacles with high resolution capability in range and velocity. Also in [17], an improved FMCW radar system is introduced by including a perfect binary sequences pairs. This system uses autocorrelation functions to determine IF (intermediate- frequency) signal, and measures distance and velocity. 
In our previous work [18], we have proposed the analytical expression of detection and false alarm probabilities and validated them by numerical simulations in different situations. Then, the ROCs performances of CFACAR (Constant False Alarm Coded Anti-collision Radar) system had been given and compared in term of used codes. We concluded that the Kasami codes of type 2 guarantee a good ROCs and many users can share the same channel like CDMA technique. But, the ROCs are bad for $S N R<=-15 d B$. To improve them in this very noisy case, we propose a new scheme version of the receiver.

This paper is organized as follows: In section II, we recall the principle of actual version of the receiver called SCR (Single Correlation Receiver). Then, we discuss the time aspect of moving a target compared to the time needed to computing of one correlation. In section III, we describe the proposed version of the receiver called Averaging Correlation Receiver (ACR). Then, in section IV ROCs numerical results of both SCR and ACR considered in different situations are given and in section $\mathrm{V}$, a conclusion is drawn.

\section{PRINCIPLE OF CFACAR}

The principle of the CFACAR system is to generate a pseudo random code $c(i)$ of length $N$ as described in the Figure 1. This pseudo random code modulates a microwave carrier in BPSK (Bi Phase Shift Keying) at $76-77 \mathrm{GHz}$ and is transmitted towards the potential targets. After reflection on an obstacle, the reflected wave is demodulated, amplified and filtered by a low pass filter. The obtained signal is sampled at transmitted code frequency and converted to a digital signal using an Analog to Digital Converter (ADC). Then, the correlation between the reference code $c(i)$ and the received signal $r(i)$ is computed. The detection unit is added to decide if the peak exceeds or not the detection threshold. The latter is adjusted to have some desired Constant False Alarm probability values. If the decision is true, the distance from the target can be deduced using the following formula:

$$
D=k \cdot \frac{c}{2 f_{h}}=k \cdot P
$$

Where $D$ is the distance to the target (in $m$ ), $f_{h}$ is the radar code frequency (in $\mathrm{Hz}$ ), $c$ is the propagation speed of light and $P=\frac{c}{2 f_{h}}$ is the distance precision.

The estimated distance is communicated to the Control Unit (CU) which decides how to accelerate or decelerate in order to maintain a safety distance between the vehicle and the target [19].

In Baseband, we can model the received signal by the following expression:

$$
r(i)=A c(i-k)+n(i)
$$

Where $k$ is the unknown delay, $A$ is the attenuation factor and $n(i)$ is the additive white gaussian noise. At the output of the correlation receiver (Figure 2) we have:

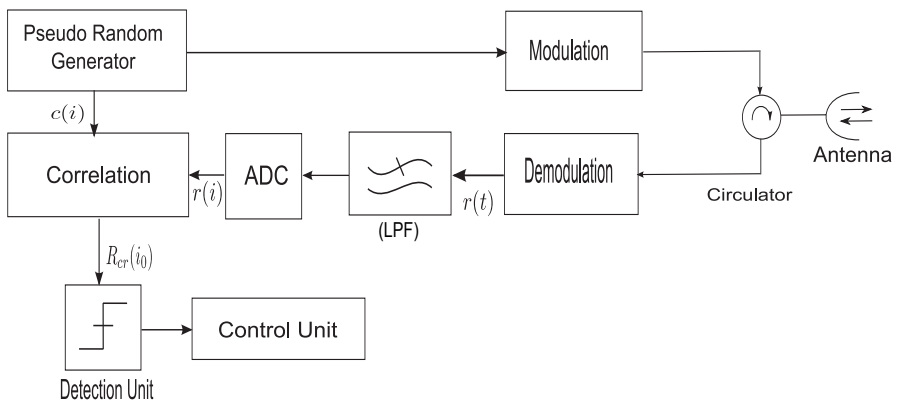

Figure 1. Coded Anti-collision Radar.

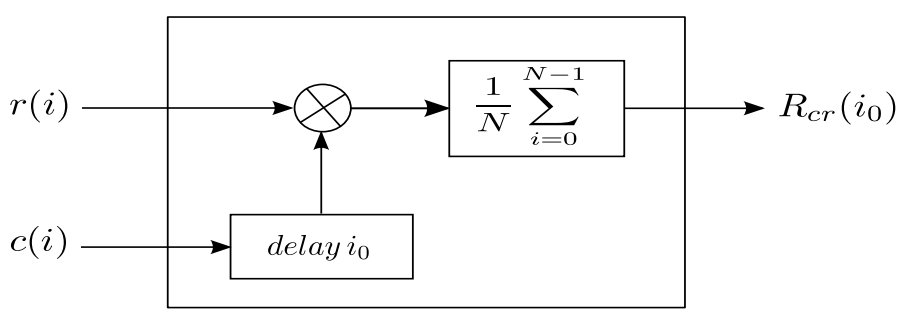

Figure 2. Single Correlation Receiver (SCR).

$$
R_{c r}\left(i_{0}\right)=\sum_{i=0}^{N-1} c\left(i-i_{0}\right) r(i)=A R_{c c}\left(i_{0}-k\right)+R_{c n}\left(i_{0}\right)
$$

Then this signal is compared to the threshold at decision time $\left(i_{0}=k\right)$. The main specification of our system is to generate the reference code and to perform computation at $100 \mathrm{MHz}$.

It is shown in [2], [20] that the SNR gain is equal to code length $N$ :

$$
G=\frac{S N R_{\text {out }}}{S N R_{\text {in }}}=N
$$

Where $S N R_{\text {in }}$ and $S N R_{\text {out }}$ are respectively input and output $S N R$ of correlation receiver.

In $d B$ this gain can be written as:

$$
G_{d B}=10 \log (G)=10 \log (N)
$$

As long as the code is the longest this gain increases. In our study we take $N=1023$ that represents the compromise between good performances and less complexity. In fact, this length ensures a high SNR gain $\left(G_{d B}=30 d B\right)$, the correlation complexity is fairly reasonable for an implementation in real time [21]. Also, if we increase the code frequency, we get a good precision in distance. The frequency of $100 \mathrm{M} \mathrm{Hz}$ give a precision in distance $P=1.5 \mathrm{~m}$ and maximum range is equal to $D_{\max }=N * P=1534 \mathrm{~m}$.

Assuming that one obstacle vehicle moves at constant relative speed $v_{r}$ compared to an other vehicle equipped by the radar (Figure 3).

The distance is expressed as:

$$
D(t)=D_{0}+v_{r}\left(t-t_{0}\right)
$$




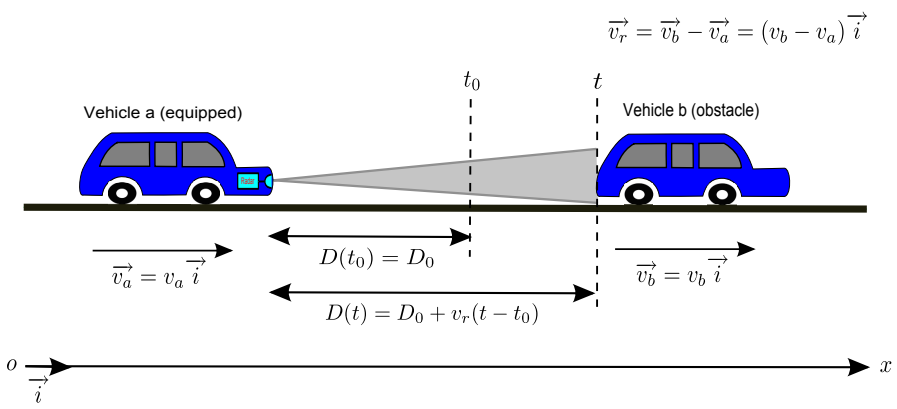

Figure 3. Displacement of obstacle vehicle compared to the vehicle equipped by the radar.

Where $D_{0}$ is the distance at instant $t=t_{0}$.

According to the sign of the velocity $v_{r}$, positive or negative, the distance may increase or decrease in time. The time of computation of the correlation is assumed, for an implementation in real time in an FPGA (Field Programmable Gates Array) based card, to be equal to the duration of one period $t_{c}=N T_{h}=N / f_{h}$. During this time, the obstacle vehicle can move by:

$$
\begin{aligned}
D_{c} & =\left|D\left(t_{c}+t_{0}\right)-D\left(t_{0}\right)\right| \\
& =\left|D_{0}+v_{r} .\left(t_{c}+t_{0}-t_{0}\right)-D_{0}\right| \\
& =\frac{N}{f_{h}}\left|v_{r}\right|
\end{aligned}
$$

This displacement is negligible compared to precision, because:

$$
\begin{aligned}
D_{c} & \ll P \\
\frac{N}{f_{h}}\left|v_{r}\right| & \ll \frac{c}{2 f_{h}} \\
\left|v_{r}\right| & \ll \frac{c}{2 N}
\end{aligned}
$$

This inequality remains valid since in the road environment, the relative speed does not exceed a hundred of $\mathrm{Km} / \mathrm{h}, \mathrm{c} / 2=$ $5.410^{8} \mathrm{Km} / \mathrm{h}$ and $N$ is in order of thousands. In our case we have $N=1023$ and $\left|v_{r}\right|$ should be negligible compared to $\frac{c}{2 N}=5.2710^{5} \mathrm{Km} / \mathrm{h}$.

We have drawn in Figure 4, this displacement $D_{c}$ expressed in $m m$ as function as $\left|v_{r}\right|$ with values $N=1023$ and $f_{h}=$ $100 \mathrm{MHz}$.

From this figure, it can be observed that the obstacle moves in the order of $0.85 \mathrm{~mm} \mathrm{~mm}$ for the maximum speed $300 \mathrm{Km} / \mathrm{h}$. This remains very negligible compared to accuracy $1.5 \mathrm{~m}$.

On the other hand, the CFACAR system does not measure the Doppler directly as FMCW radar does. But, it determines the speed following the variations in the distance over time. This speed is determined by the ratio between the estimated distances between two times $t_{1}$ and $t_{2}>t_{1}$ on the elapsed time between these two instants:

$$
v_{r}=\frac{D\left(t_{2}\right)-D\left(t_{1}\right)}{t_{2}-t_{1}} \quad t_{2}>t_{1}
$$

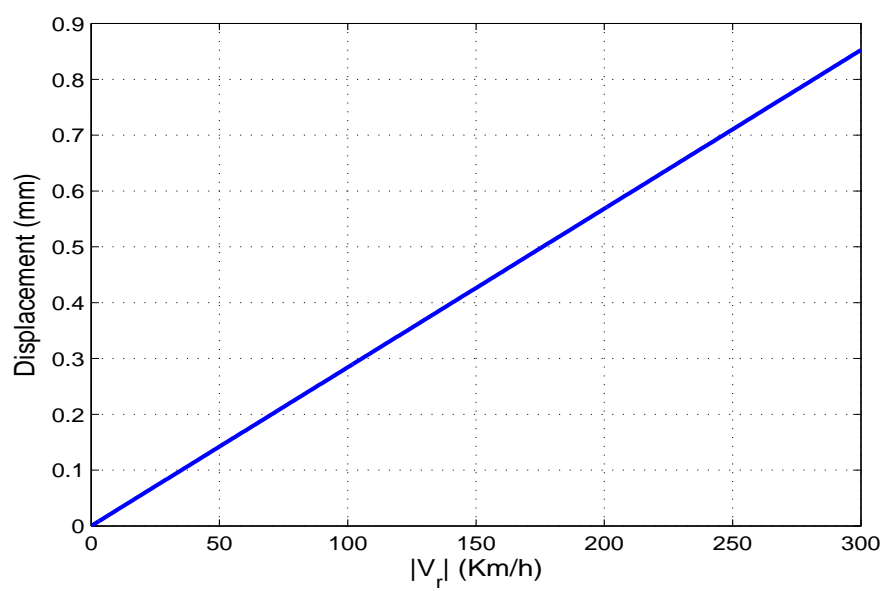

Figure 4. Displacement of the obstacle vehicle during computation time.

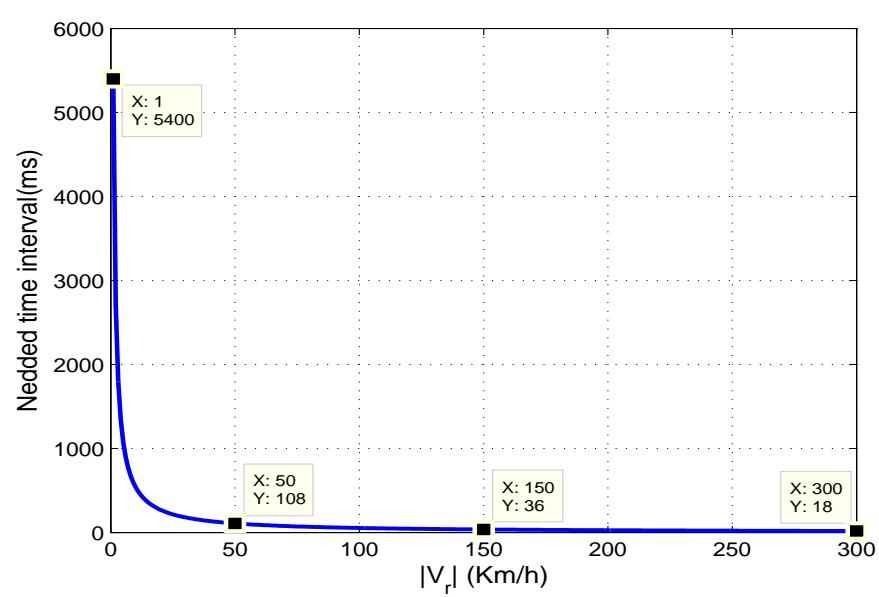

Figure 5. Time needed to have a change of distance equal to the precision.

As the vehicle moves at a distance negligible compared to the precision $\left(D\left(t_{2}\right) \approx D\left(t_{1}\right)\right)$, between the two instants $t_{1}$ and $t_{2}$, the speed $v_{r} \approx 0$. To estimate the velocity we need some minimum time. We note $\Delta t=t_{2}-t_{1}$ this time is so chosen to get a change in distance that is equal to the distance accuracy:

$$
\Delta t=t_{2}-t_{1}=\frac{P}{v_{r}}=\frac{c}{2 f_{h} v_{r}}
$$

But this time depends on the speed itself, so to get an idea on its variations, we deliberately plotted them in Figure 5.

On this figure, it can be observed that there's a huge gap between the possible values. We must take this time in the order of several seconds for lower speeds and in order of tens of milliseconds for high speeds.

For distance, we can re-estimate it at any instant in $\Delta t$ interval. Then, we have a time to estimate distance which is not necessary at each small interval of actual processing $\left(t_{c}=\right.$ $\left.N / f_{h}=10.23 \mu s\right)$.

For velocity, we must take a minimum interval equal to $\Delta t$. For example, if the actual value is $\left|v_{r}\right|=150 \mathrm{Km} / \mathrm{h}$ the minimum is an interval of $36 \mathrm{~ms}$ (otherwise, for inferior 
interval this computation is not needed because $\left|v_{r}\right| \approx 0$ and if we apply the formula of equation 10, we obtain a worst result $\left.\left(\left|v_{r}\right|=1.5 \mathrm{~m} / 10.23 \mu \mathrm{s}=52710^{3} \mathrm{Km} / \mathrm{h}\right)\right)$. This a disadvantage of our system.

In [18], for SCR receiver, the expressions of probabilities are derived for three cases:

- case 1: one user with one target, the detection probability of target is:

$$
P_{D}=\frac{1}{2} \operatorname{erfc}\left[\sqrt{\frac{N}{2}}\left(\frac{Y_{0}}{\sigma_{n}}-\sqrt{S N R}\right]\right.
$$

Where:

- $r(i)=A c(i-k)+n(i)$ is the recived signal model.

- $A$ and $k$ are the attenuation and delay of signal provided from the target.

- $c(i)$ is the code associated to user.

- $S N R=A^{2} / \sigma_{n}^{2}$ is the input SNR.

- case 2: one user with multi-targets, the detection probability of $j^{\text {th }}$ target is:

$$
P_{D_{j}}=\frac{1}{2} \operatorname{erfc}\left[\sqrt{\frac{N}{2}}\left(\frac{Y_{0}-B_{j}}{\sigma_{n}}-\sqrt{S N R_{j}}\right)\right]
$$

Where:

- $r(i)=\sum_{l=1}^{N_{t}} A_{l} c\left(i-k_{l}\right)+n(i)$ is the received signal model.

- $N_{t}$ is the number of targets.

- $A_{l}$ and $k_{l}$ are the attenuation and delay coefficients of echo signal of $l^{\text {th }}$ target.

- $c(i)$ is the code associated to user.

- $S N R_{j}=A_{j}^{2} / \sigma_{n}^{2}$ is the input SNR of $j^{t h}$ target.

- $B_{j}=\sum_{l=1, l \neq j}^{N_{t}} A_{l} R_{c c}\left(k_{j}-k_{l}\right)$ is the interference factor between targets.

- case 3: multi-users, the detection probability of target of radar $m$ :

$$
P_{D_{m}}=\frac{1}{2} \operatorname{erfc}\left[\sqrt{\frac{N}{2}}\left(\frac{Y_{0}-B_{m}}{\sigma_{n}}-\sqrt{S N R_{m}}\right)\right]
$$

Where:

- $r_{m}(i)=\sum_{j=1}^{N_{u}} A_{j} c_{j}\left(i-k_{j}\right)+n(i)$ is the received signal model of $m^{\text {th }}$ user.

- $N_{u}$ is the number of users,

- $A_{j}$ and $k_{j}$ are the attenuation and delay of signal provided from the $j^{\text {th }}$ user.

- $S N R_{m}=A_{m}^{2} / \sigma_{n}^{2}$ is the input SNR of $m^{t h}$ user.

- $B_{m}=\sum_{j=1, j \neq m}^{N_{u}} A_{j} R_{c_{m} c_{j}}\left(k_{m}-k_{j}\right)$ is the interference factor between users.

- $c_{m}(i)$ is the code associated to $m^{t h}$ user.

- False alarm probability for the three cases:

$$
P_{F A}=\frac{1}{2} \operatorname{erfc}\left(\sqrt{\frac{N}{2}} \frac{Y_{0}}{\sigma_{n}}\right)
$$

For all probabilities, $Y_{0}$ is the detection threshold, $\sigma_{n}$ is the noise variance and $\operatorname{erfc}(x)$ is the complementary error function defined as: $\operatorname{erf} c(x)=\frac{2}{\sqrt{\pi}} \int_{x}^{+\infty} e^{-t^{2}} d t$.

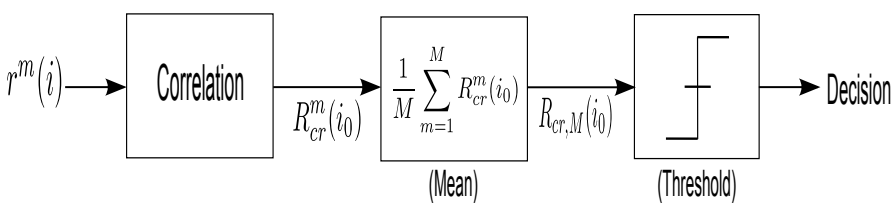

Figure 6. Averaging Correlation Receiver (ACR).

\section{IMPROVING DETECTION BY AVERAGING}

The performances of SCR receiver depend directly upon the ability to detect a peak at decision instant of the output signal. We saw in [18] that the ROCs for $S N R$ less than $-15 d B$ are not perfect due to large fluctuation of the output noise with respect to the average of the signal at the decision instant. As discussed previously, the system have a time to estimate the distance. So, we propose to take an average of the consecutive correlation results (Averaging Correlation Receiver, ACR) as shown in Figure 6. We denote the received signal by $r^{m}(i)$ and its correlation with the reference code $R_{c r}^{m}\left(i_{0}\right)$ at $m^{t h}$ realization in which we have $N$ samples for both signals. The average of $M$ last correlations gives the output signal:

$$
R_{c r, M}\left(i_{0}\right)=\frac{1}{M} \sum_{m=1}^{M} R_{c r}^{m}\left(i_{0}\right)
$$

Then, we have for:

- case 1: in the single-user case with a single target, at decision instant we have ar random variable $R_{c r}^{m}(k)=$ $Y_{k}^{m}$, for a $m^{t h}$ realization, that is of type Gaussian with a mean equal to $A$ and variance equal to $\sigma_{Y_{k}^{m}}^{2}=$ $\sigma_{Y_{n k}}^{2}=\sigma_{n}^{2} / N$ [18]. We assume that all the variables in the last $M$ realizations are independent, then the output of the correlation average $Y_{M}=\frac{1}{M} \sum_{m=1}^{M} Y_{k}^{m}$ is a Gaussian random variable with same average $A$ and variance $\sigma_{Y_{M}}^{2}=\frac{\sigma_{Y_{k}^{m}}^{2}}{M}=\frac{\sigma_{n}^{2}}{N . M}$ (Law of the sum of independent Gaussian random variables [22]). In outside the decision instant (output noise) of the output signal is a zero-mean Gaussian random variable with the same variance as the decision instant one. When $M$ is greater, the fluctuation of the output noise will be reduced (i.e. its variance decreases).

- case 2 and 3: in the case of single user with multiple targets and multi-user case, the variances of signal at and outside decision instant does not change, while the average at decision instant changes as function of used codes and delays (parameters $B_{j}$ and $B_{m}$ ).

Then, by analogy with demonstrations for SCR in [18], we deduce the expressions of $P_{D}$ and $P_{F A}$ for ACR by just replacing $N$ per $M N$ in equations $11,12,13$ and 14 :

- case 1: the detection probability of target is:

$$
P_{D}=\frac{1}{2} \operatorname{erfc}\left[\sqrt{\frac{M N}{2}}\left(\frac{Y_{0}}{\sigma_{n}}-\sqrt{S N R}\right)\right]
$$

- case 2: the detection probability of $j^{\text {th }}$ target is: 
Table I

REQUIRED $M$ FOR ALL CASES

\begin{tabular}{|c|c|}
\hline$S N R$ & required $M$ \\
\hline$-15 d B$ & 1 \\
\hline$-20 d B$ & 3 \\
\hline$-30 d B$ & 30 \\
\hline$-40 d B$ & 400 \\
\hline
\end{tabular}

$$
P_{D_{j}}=\frac{1}{2} \operatorname{erfc}\left[\sqrt{\frac{M N}{2}}\left(\frac{Y_{0}-B_{j}}{\sigma_{n}}-\sqrt{S N R_{j}}\right)\right]
$$

- case3: the detection probability of target of radar $m$ :

$$
P_{D_{m}}=\frac{1}{2} \operatorname{erfc}\left[\sqrt{\frac{M N}{2}}\left(\frac{Y_{0}-B_{m}}{\sigma_{n}}-\sqrt{S N R_{m}}\right)\right]
$$

- False alarm probability for the three cases:

$$
P_{F A}=\frac{1}{2} \operatorname{erfc}\left(\sqrt{\frac{M N}{2}} \frac{Y_{0}}{\sigma_{n}}\right)
$$

\section{NUMERICAL RESULTS}

In this section, we use the Kasami codes of type 2 which are the best codes that can be used as discussed in [18]. To see the improvement made by averaging, we present, in Figure 7, an example of one realization under hypothesis $H_{1}$ (presence of targets) for different values of $M$ with three targets at $\left(k_{1}=\right.$ $\left.100, k_{2}=200, k_{3}=300\right)$ and a $S N R_{j}=-30 d B(\mathrm{SNR}$ of each target), $j=1,2,3$. The thresholds are set to have a $P_{F A}=10^{-5}$ using the threshold expression deduced from equation (19):

$$
Y_{0}=\sqrt{\frac{2}{M . N}} \operatorname{erfc}^{-1}\left(2 P_{F A}\right) \sigma_{n}
$$

We note that, for $M=1$, it is impossible to detect any target, for $M=20$, we detect only two targets, and for $M=$ 30 and $M=50$, the three targets are detected. As we increase $M$, the detection comes more better.

We plot the ROCs for different $S N R$ and $M$ for the three cases in Figures 8, 9, 10,11 and 12.

We have approximatively the same remarks for all cases. We note that, where $M$ increases, the ROCs become perfect. The required length $M$, to have the good ROCs, is reported in Table I. The corresponding values of $\left(P_{D}\right)$ to have a $P_{F A}=10^{-5}$ are always greater than 0.9 for all cases with a little difference between them.

According to equation (6), we have at instant $t_{0}, D\left(t_{0}\right)=$ $D_{0}$ and at instant $t_{1}=t_{0}+t_{c}$, we have $D\left(t_{1}\right)=D_{0}+v_{r} . t_{c}$, where $t_{c}$ is the computation time of averaging of later $M$ correlations, and if we assume that each correlation takes $N . T_{h}=N / F_{h}$ for an implementation in real time. So this time is approximatively $t_{c}=(M . N) / F_{h}$. We must have, to use the averaging, an negligible variation of distance compared to precision $P$ between the two instants $t_{0}$ and $t_{1}$ $\left(\left|D\left(t_{1}\right)-D\left(t_{0}\right)\right| \ll P\right)$. This is equivalent to assumption that
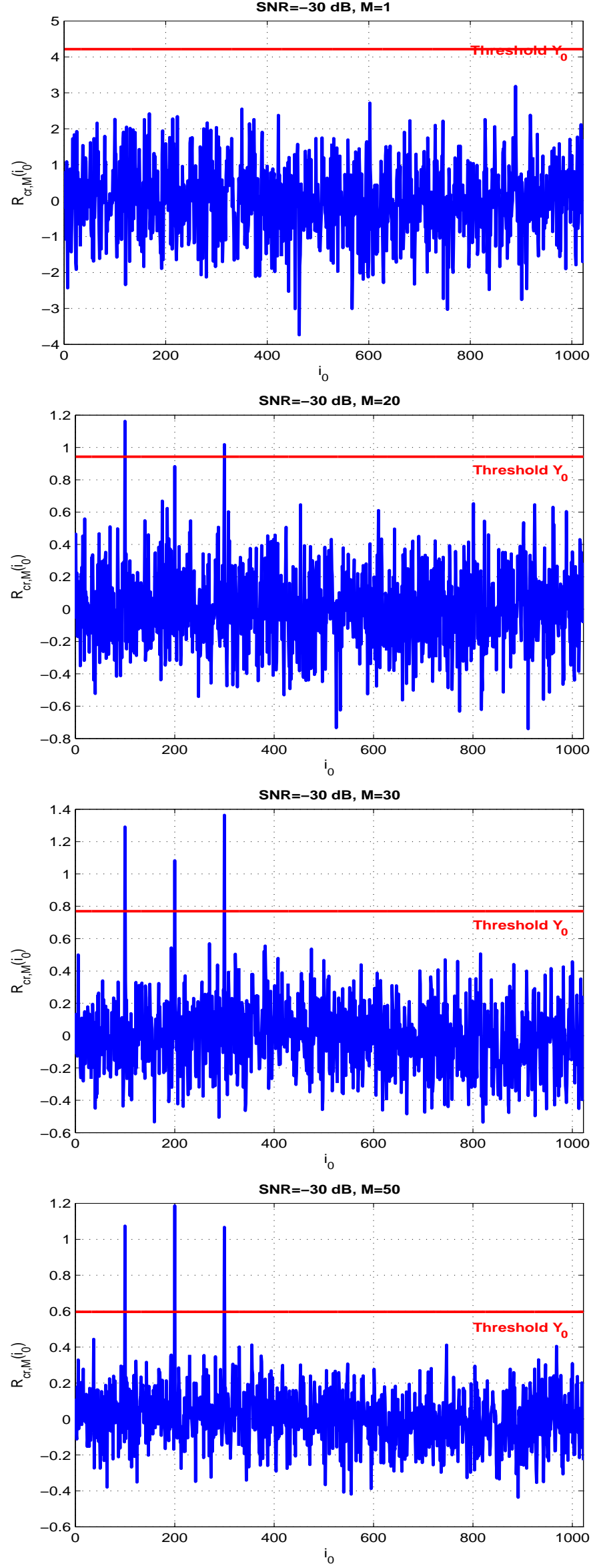

Figure 7. Examples of realizations in the mono-user case with three targets and $S N R=-30 \mathrm{~dB}$. 
$\mathrm{SNR}=-15 \mathrm{~dB}$

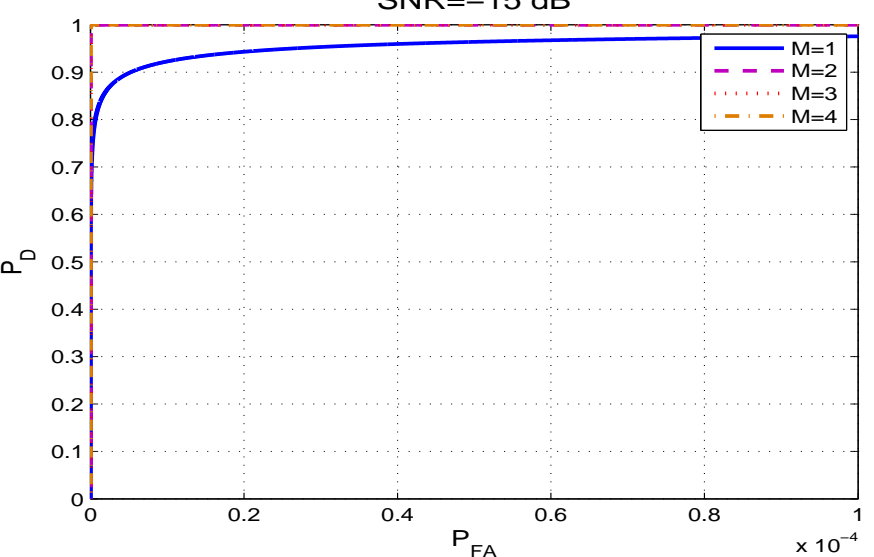

$\mathrm{SNR}=-20 \mathrm{~dB}$

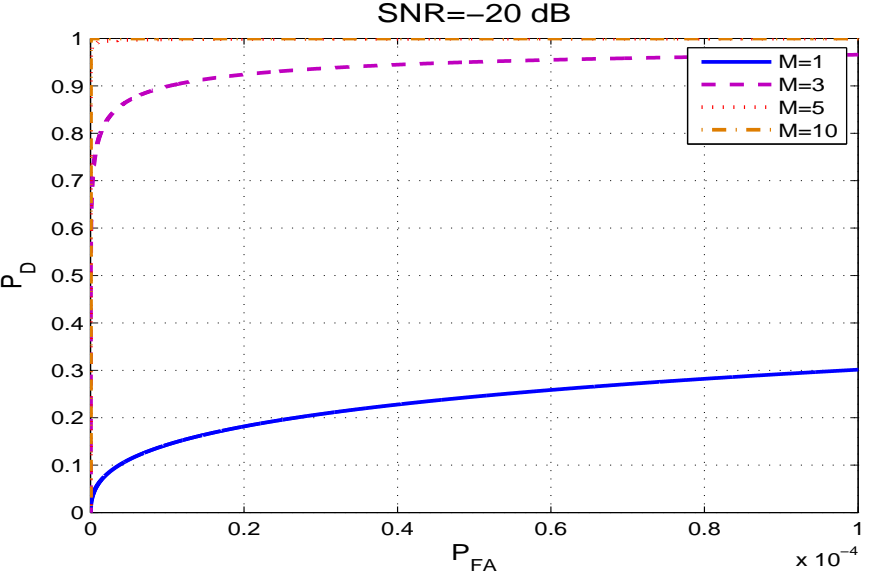

$S N R=-30 \mathrm{~dB}$

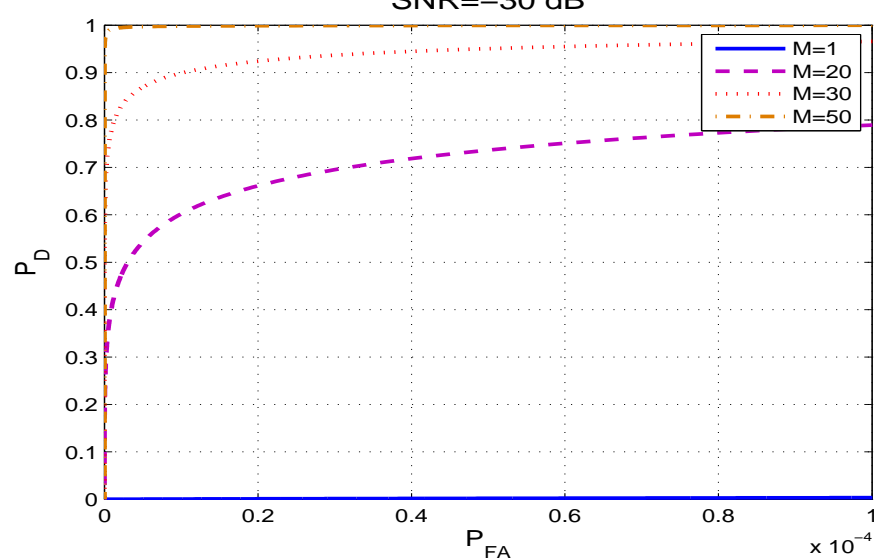

$S N R=-40 \mathrm{~dB}$

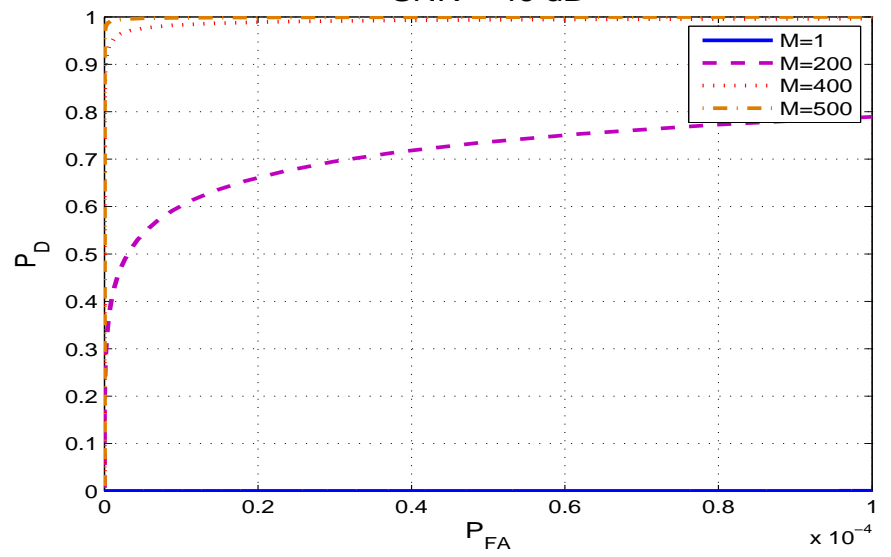

$\mathrm{SNR}=-15 \mathrm{~dB}$

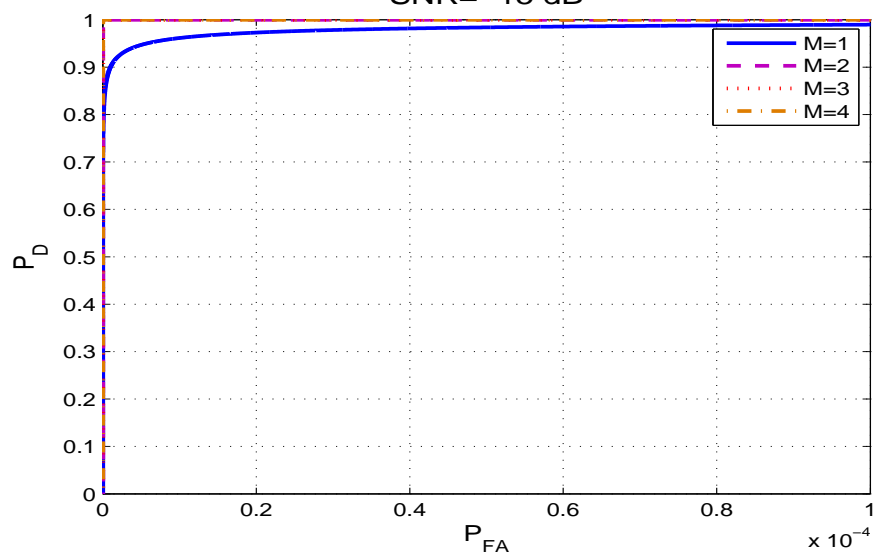

$\mathrm{SNR}=-20 \mathrm{~dB}$

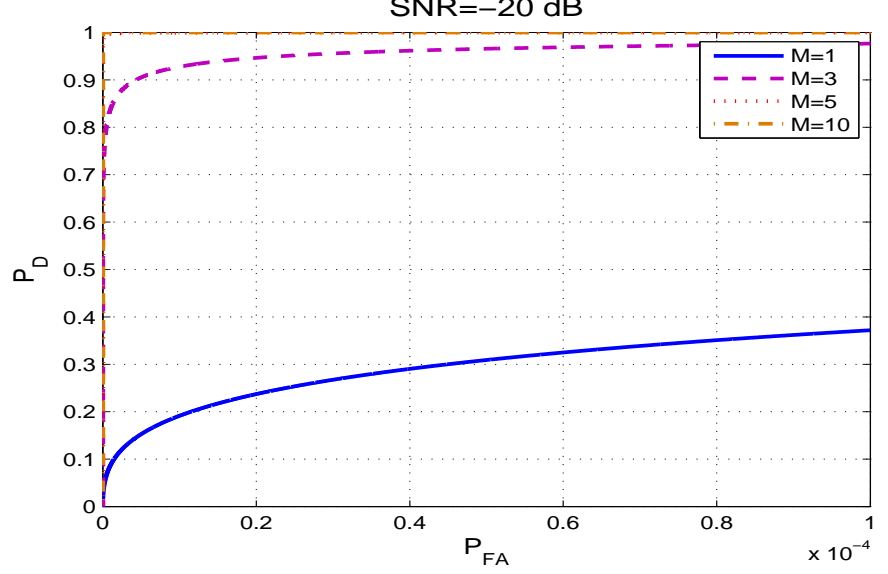

$S N R=-30 \mathrm{~dB}$

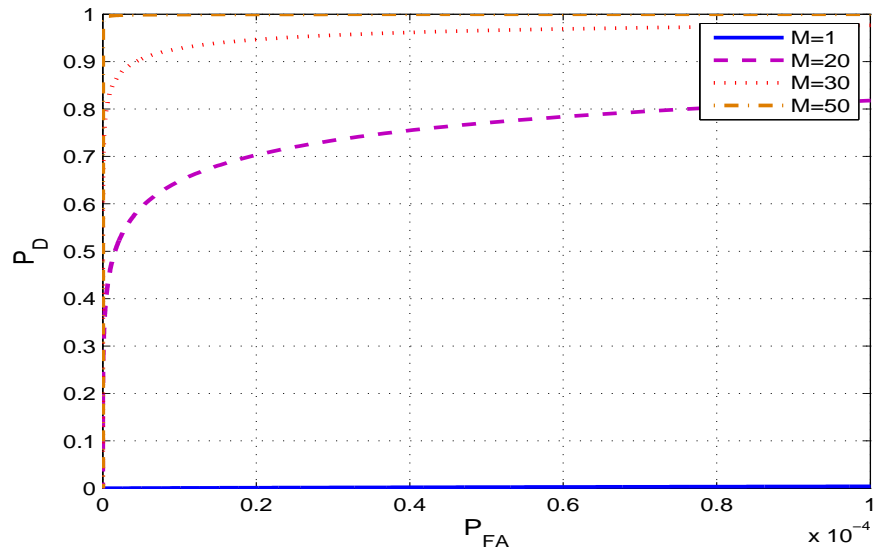

$S N R=-40 \mathrm{~dB}$

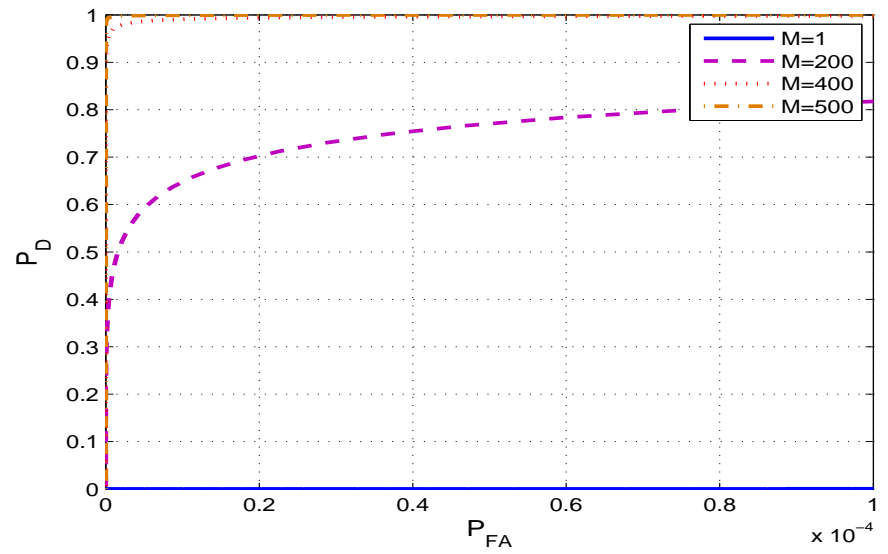

Figure 9. ROCs of the $1^{\text {st }}$ target in second case. 
$\mathrm{SNR}=-15 \mathrm{~dB}$

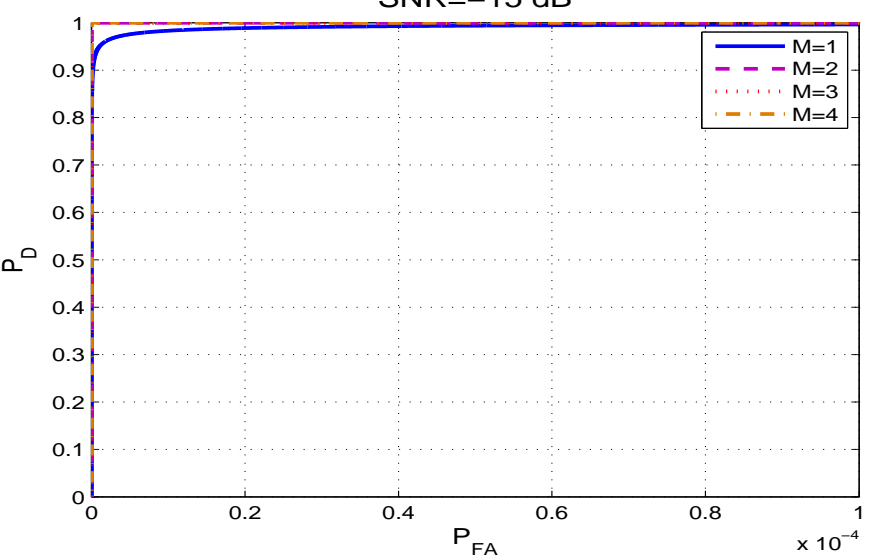

$S N R=-20 \mathrm{~dB}$

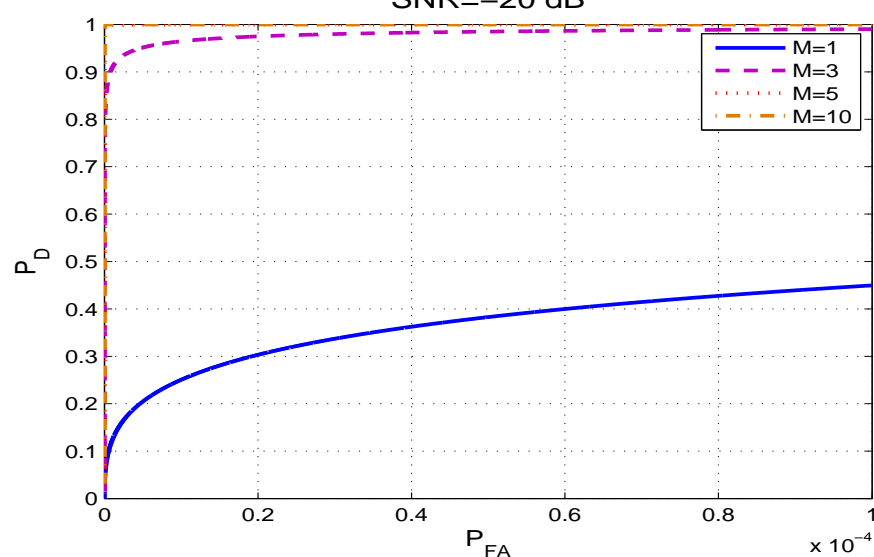

$S N R=-30 d B$

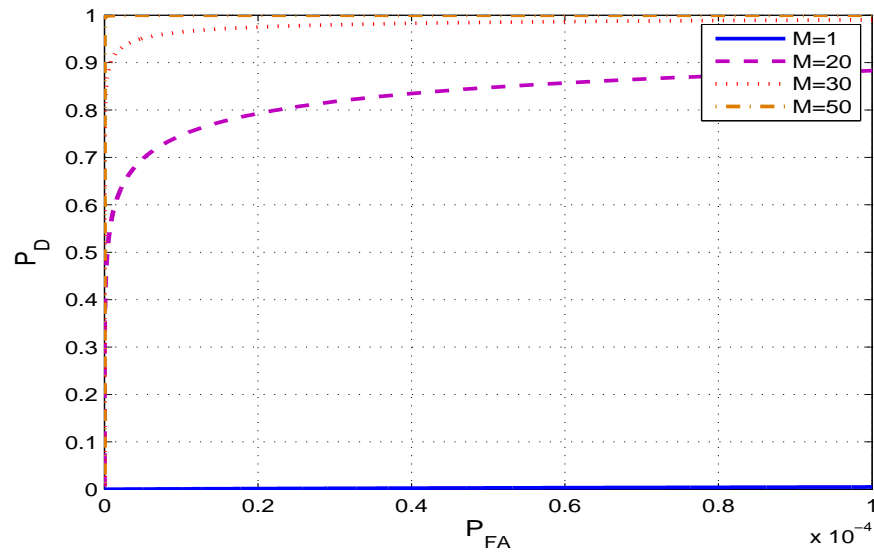

$S N R=-40 \mathrm{~dB}$

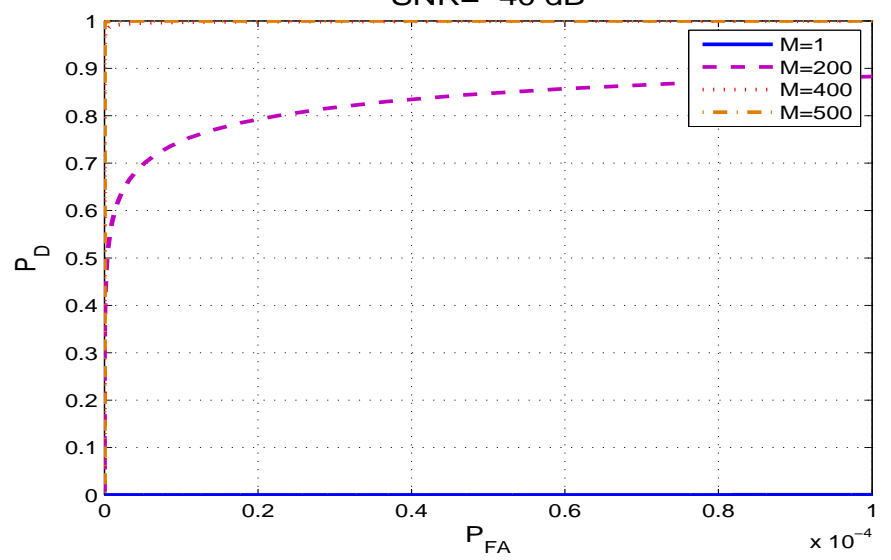

$\mathrm{SNR}=-15 \mathrm{~dB}$

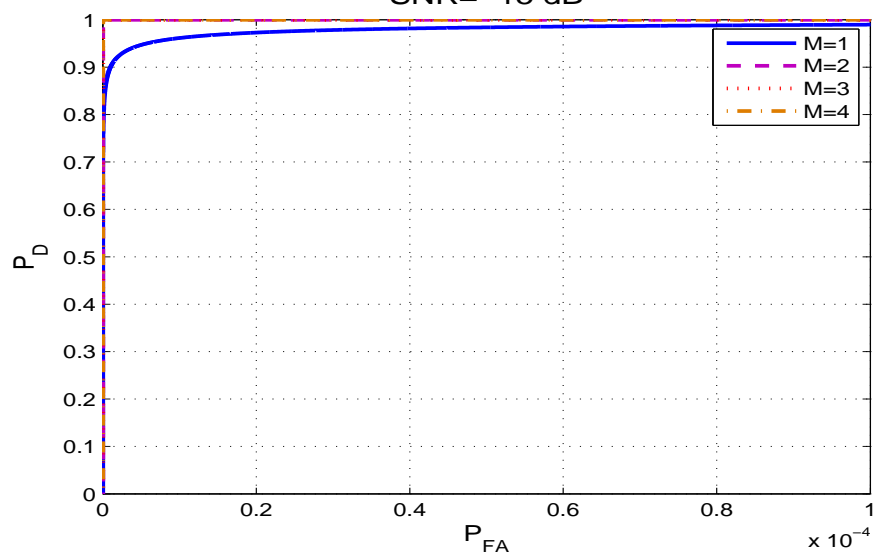

$\mathrm{SNR}=-20 \mathrm{~dB}$

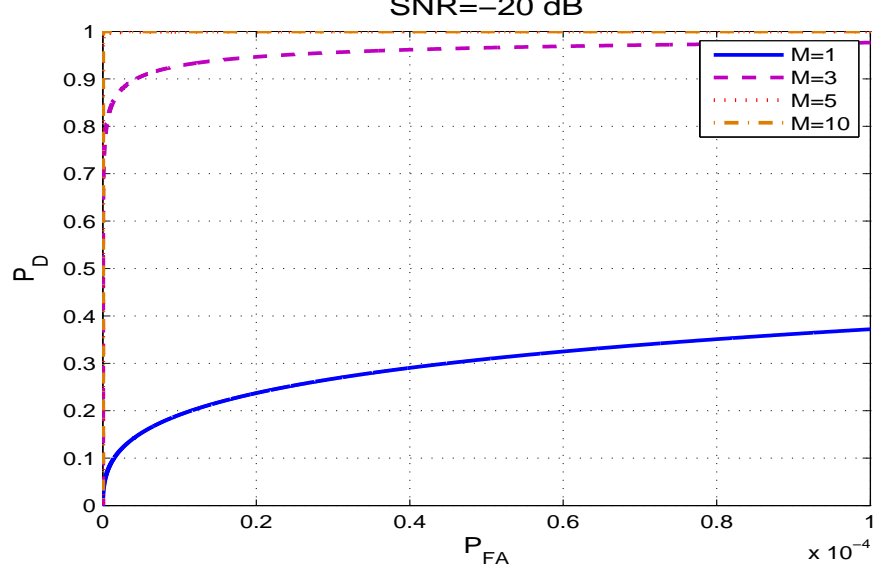

$S N R=-30 \mathrm{~dB}$

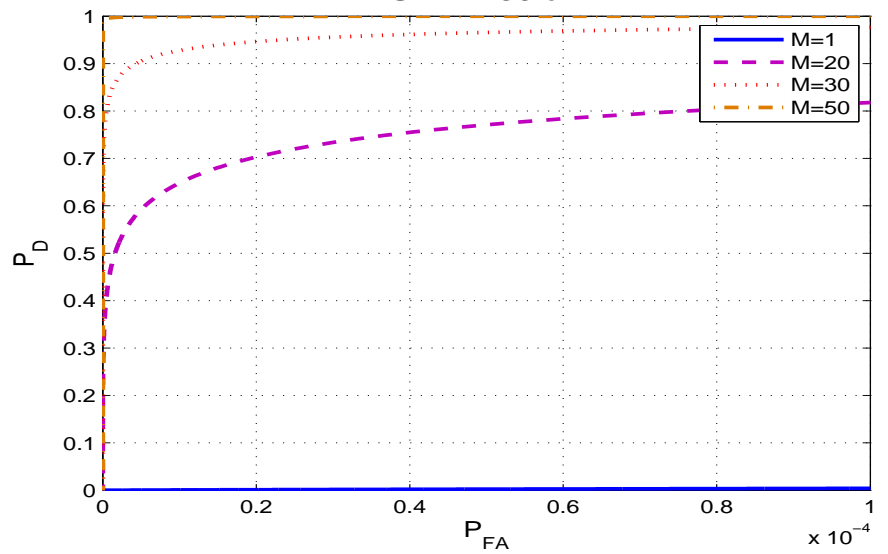

$S N R=-40 \mathrm{~dB}$

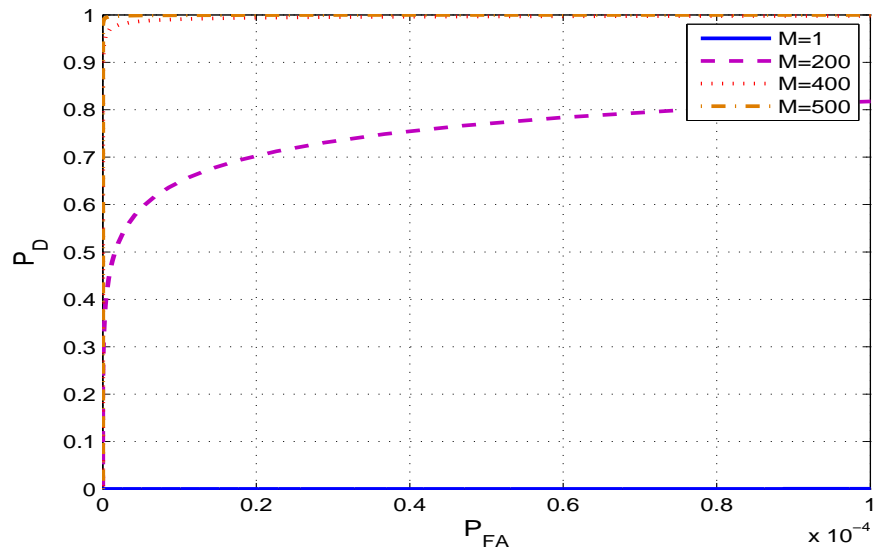

Figure 11. ROCs of the $3^{\text {ed }}$ target in second case.

Figure 10. ROCs of the $2^{\text {ed }}$ target in second case. 

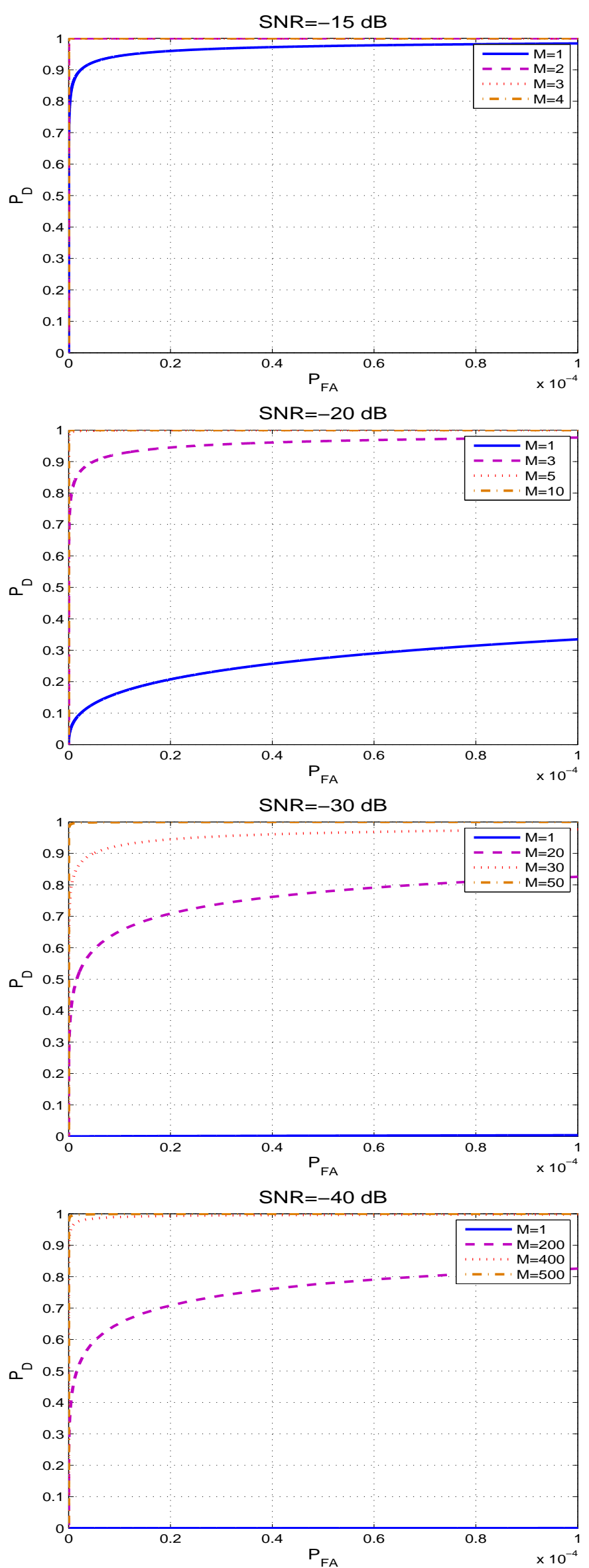

Figure 12. ROCs in third case.

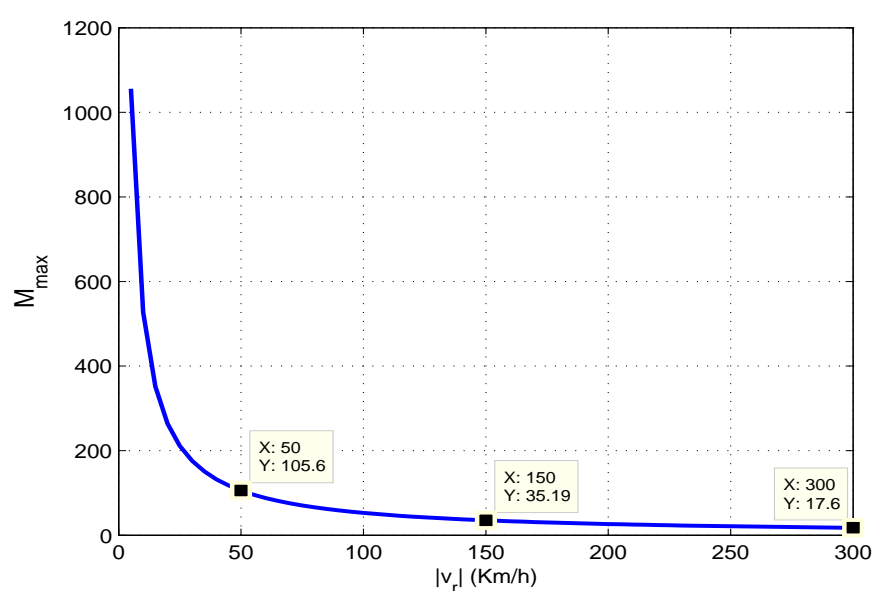

Figure 13. $M_{\max }$ as fuction as $\left|v_{r}\right|$.

the channel does not change in this interval. We note $\eta$ a small number used to ensure this last condition:

$$
\begin{aligned}
\left|D\left(t_{1}\right)-D\left(t_{0}\right)\right| & \leq \eta \cdot P \\
\left|v_{r} \cdot t_{c}\right|=\left|\frac{v_{r} \cdot M \cdot N}{F_{h}}\right| & \leq \frac{\eta \cdot c}{2 \cdot F_{h}} \\
M & \leq \frac{\eta \cdot c}{2 \cdot N \cdot\left|v_{r}\right|} \\
M & \leq M_{\max }
\end{aligned}
$$

Then, the maximum value that can take $M$ is:

$$
M_{\max }=\frac{\eta \cdot c}{2 . N \cdot\left|v_{r}\right|}
$$

This maximum is inversely proportional to the relative speed $v_{r}$. As this speed is low we have a large $M_{\max }$ that guarantees a good detection until very small $S N R$ and for high value of speed we can have a good detection until some value of $S N R$. To have an idea about the variation of $M_{\max }$ as function as $\left|v_{r}\right|$, we plotted it in Figure 13 for $\eta=1 \%$. For example and according to table I, we can guarantee for $\left|v_{r}\right|=150 \mathrm{Km} / \mathrm{h}$ and $M_{\max }=35$ a good detection until a $S N R=-30 \mathrm{~dB}$ and for $\left|v_{r}\right|=300 \mathrm{Km} / \mathrm{h}$ (the worst case) and $M_{\max }=17$ until a $S N R=-27 d B$ approximatively.

We plot in Figure $14 P_{D}$ as function of SNR for case 1 with fixed $P_{F A}=10^{-5}$ and different values of $M$. The other cases give approximatively the same results in terms of $P_{D}$.

As $S N R$ decreases we must increase $M$ but increasing $M$ is not possible for large value values of velocity. For example for $300 \mathrm{Km} / \mathrm{h}$ we can have a good detection $\left(P_{D} \geq 0.9\right)$ from a minimum $S N R=-15 d B$ otherwise for $5 \mathrm{Km} / \mathrm{h}$ we can have a good detection $\left(P_{D} \geq 0.9\right)$ from a minimum $S N R=-45 \mathrm{~dB}$. We can modify the receiver by adding some blocks to determine $M=M_{\max }$ adaptively by using the equation (21) and the estimation of $v_{r}$, Figure 15. But this version does not match with our system as it is not able to goodly estimate the velocity. To get a good estimation of the velocity using our system or get a better estimation than ours, we propose to combine this sensor with other techniques like 


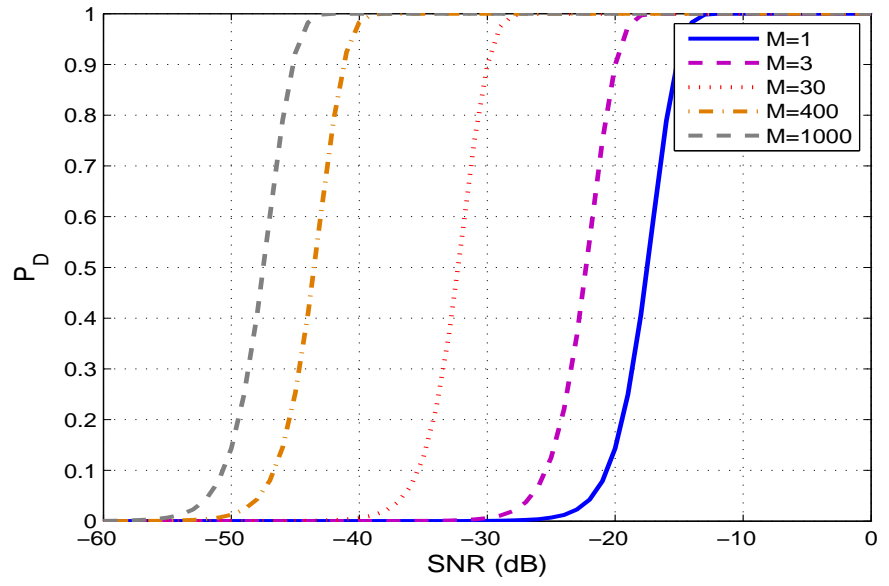

Figure 14. $P_{D}$ as function as SNR.

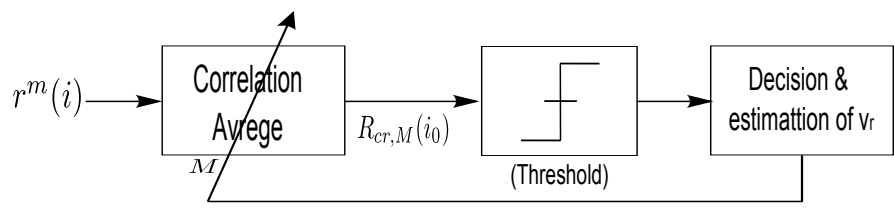

Figure 15. Adaptive averaging correlation receiver.

FMCW and MFSK [23], [24], [25]. This can be done as a future improvement in future work.

\section{CONCLUSION}

We have proposed in previous works the expression of detection and false alarm probabilities for CFACAR. We treated three cases and plotted the ROCs for each one. We observed that these latter are very bad for low SNR (inferior to $-15 d B$ ) and good for high SNR. To improve the performances in very low SNR cases, we propose in this paper, the ACR receiver which consists in computing the average of the $M$ later correlation results. This is possible because the obstacle does not move enough compared to precision of the system. Then, the expressions of detection and false alarm probabilities are derived and then the new ROCs are plotted. We can ensure a good detection in very noisy case like the situation where we have a $S N R=-40 \mathrm{~dB}$ by choosing a suitable value of length of ACR. This length depends on relative velocity and varies between a large value for low velocity and low value for high velocity. For examples, for a velocity of $5 \mathrm{Km} / \mathrm{h}$ with $M=1055$, we can go down until $S N R=-45 d B$ and for a velocity of $300 \mathrm{Km} / \mathrm{h}$ with $M=17$, we can go down until $S N R=-27 \mathrm{~dB}$. We propose to choose the length adaptively. But we need a good estimation of velocity that is not ensured by our sensor. Our future work will be focused on finding a way to get a good estimation of velocity by using other techniques like FMCW while maintaining the CDMA characteristics of the potential new system.

\section{REFERENCES}

[1] P. Seiler, B. Song, and J. K. Hedrick, "Development of a collision avoidance system," Development, vol. 4, pp. 17-22, 1998.

[2] A.Menhaj, "Études de systèmes anti-collision basés sur les techniques radar pour véhicules routiers," PhD thesis, University of Valenciennes (In French), December 1996.
[3] J.Assaad, A. Menhaj, N. Haese, and C. Bruneel, "Signal processing study for an fmcw collision avoidance radar system," Signal processing, vol. 61, $n^{\circ} 1$, pp. 24-33, Aout 1997

[4] B.Frémont, A.Menhaj, P.Deloof, and M.Heddebaut, "A cooperative collision avoidance and communication system for railway transports," $3 r d$ IEEE Conference on intelligent transportation systems, Dearborn, October 2000.

[5] J. M. Allebach, R. M. Narayanan, B. Hoe, and S. P. Broderick, "Bistatic and multistatic target identification for through-wall radar imaging," in SPIE Defense + Security, pp. 94610G-94610G, International Society for Optics and Photonics, 2015.

[6] I. Cohen, R. Elster, and N. Levanon, "Good practical continuous waveform for active bistatic radar," IET Radar, Sonar \& Navigation, 2015.

[7] L. Anitori, M. Otten, W. van Rossum, A. Maleki, and R. Baraniuk, "Compressive cfar radar detection," in Radar Conference (RADAR), 2012 IEEE, pp. 0320-0325, May 2012.

[8] J.-J. Lin, Y.-P. Li, W.-C. Hsu, and T.-S. Lee, "Design of an fmcw radar baseband signal processing system for automotive application," SpringerPlus, vol. 5, no. 1, pp. 1-16, 2016.

[9] Y. Kwon, R. Narayanan, and M. Rangaswamy, "Multi-target detection using total correlation for noise radar systems," Aerospace and Electronic Systems, IEEE Transactions on, vol. 49, pp. 1251-1262, APRIL 2013.

[10] C. Fischer, H. L. Blocher, J. Dickmann, and W. Menzel, "Robust detection and mitigation of mutual interference in automotive radar," in Radar Symposium (IRS), 2015 16th International, pp. 143-148, IEEE, 2015.

[11] S. Li and G. Bi, "Improved spectrum sensing method for cognitive radio based on time domain averaging and correlation," in The Proceedings of the Second International Conference on Communications, Signal Processing, and Systems, pp. 1229-1237, Springer, 2014.

[12] C.-P. Lai and R.M.Narayanan, "Ultrawideband random noise radar design for through-wall surveillance," Aerospace and Electronic Systems, IEEE Transactions on, vol. 46, no. 4, pp. 1716-1730, 2010.

[13] Z. Zhang, Z. Lu, Q. Chen, X. Yan, and andLi Rong Zheng, "Code division multiple access/pulse position modulation ultra-wideband radio frequency identification for internet of things: concept and analysis,' International Journal of Communication Systems, vol. 25, pp. 11031121 , September 2012.

[14] E. Takeuchi, A. Elfes, and J. Roberts, "Localization and place recognition using an ultra-wide band (uwb) radar," Field and Service Robotics, vol. 105 , pp. $275-288,2015$.

[15] M.Andres, W.Menzel, H.L.Bloecher, and J.Dickmann, "Detection of slow moving targets using automotive radar sensors," Microwave Conference $(\mathrm{GeMiC}), 2012$ The 7th German, pp. 1-4, 2012.

[16] F. Muller, S. Benesch, D. Steinbuch, T. Walter, and R. Weigel, "Mitigation of weak targets' masking due to nonlinearities in chirp sequence fmcw automotive radar at $77 \mathrm{ghz}$," in 2015 Asia-Pacific Microwave Conference (APMC), vol. 3, pp. 1-3, IEEE, 2015.

[17] J. Zhang and R. Bai, "Research of fmcw for perfect binary sequences pairs," Advances in Mechanical and Electronic Engineering, vol. 178 , pp. 21-27, 2013

[18] J. Zaidouni, A. Rivenq-Menhaj, K. Ghoumid, A. El Moussati, and P. Deloof, "Roc performances of constant false alarm coded anticollision radar in multi-user environment," International Journal of Wireless Information Networks, vol. 22, no. 4, pp. 369-385, 2015.

[19] X. Yin and M. Wang, "Research on safety distance mathematical model of pro-active head restraint in rear-end collision avoidance system," International Journal of Security and Its Applications, vol. 9, no. 1, pp. 347-356, 2015.

[20] L.Sakkila, P.Deloof, Y.Elhillali, A.Rivenq, and S.Niar, "A real time signal processing for an anticollision road radar system," Vehicular Technology Conference, 2006. VTC-2006 Fall. 2006 IEEE 64th, pp. 1-5, September 2006.

[21] L. Douadi, P. Deloof, and Y. Elhillali, "Real time implementation of reconfigurable correlation radar for road anticollision system," in Industrial Technology, 2008. ICIT 2008. IEEE International Conference on, pp. 1-7, IEEE, 2008.

[22] P. traduit par J. Maurein, "Probabilités, analyse fréquentielle, information, théorie du radar," Eyrolles, Paris, 1980.

[23] S.-H. Jeong, H.-Y. Yu, J.-E. Lee, J.-N. Oh, and K.-H. Lee, "A multibeam and multi-range radar with fmcw and digital beam forming for automotive applications," Progress In Electromagnetics Research, vol. 124 , pp. $285-299,2012$.

[24] H. Rohling and C. Möller, "Radar waveform for automotive radar systems and applications," in Radar Conference, 2008. RADAR'08. IEEE, pp. 1-4, IEEE, 2008.

[25] B. Li and A. P. Petropulu, "Distributed mimo radar based on sparse sensing: Analysis and efficient implementation," Aerospace and Electronic Systems, IEEE Transactions on, vol. 51, no. 4, pp. 3055-3070, 2015. 